\title{
CUTANEOUS MANIFESTATION OF CARDIOVASCULAR, RENAL AND MALIGNANT DISEASES
}

\author{
Manabendra Nayak ${ }^{1}$, Rahul Nayak ${ }^{2}$
}

1Postgraduate Teacher, Department of Medicine, National Board of Examination, Senior Consultant Dept. of Medicine, Down Town Hospital, Guwahati.

${ }^{2}$ Assistant Professor, Department of Microbiology, Assam Down Town University.

ABSTRACT

\section{BACKGROUND}

In clinical practice, sometimes it becomes very difficult to diagnoses when patient present with some cutaneous manifestation without definitive sing and symptoms. Therefore, it is important to know and study the different type of disease which produces skin problem. Different cardiovascular disease, metabolic disease, malignant disease and autoimmune disease may produce some exceptional dermatological problem. Sometimes skin lesion itself confuse with primary dermatological disorder. That's why it's important to know the various cutaneous manifestation of internal disease.

\section{KEYWORDS}

Cutaneous Manifestation, Renal Disease, Malignancy.

HOW TO CITE THIS ARTICLE: Nayak M, Nayak R. Cutaneous manifestation of cardiovascular, renal and malignant diseases. J. Evolution Med. Dent. Sci. 2017;6(1):62-66, DOI: 10.14260/Jemds/2017/16

\section{BACKGROUND}

There are many internal diseases that present with cutaneous manifestations. These cutaneous signs may proceed, occur concurrently or follow the onset of the internal condition. Pruritus and vasculitis are common cutaneous presentations where an underlying systemic disease may be present. Certain chronic diseases may present with distinctive skin findings, which need to be recognized to institute a search for underlying neoplasm. The skin has the potential to provide a window into the patient and aid in the diagnosis of diseases of all organ systems. ${ }^{1,2}$

\section{Cutaneous Manifestations of Cardiovascular Disease Infective Endocarditis}

Infective endocarditis results from the proliferation of microorganisms in the heart. The infection most frequently involves the heart valves, both native and prosthetic; but may also occur on the ventricular septum and on intra cardiac devices. The important lesion is the vegetation, a mass, which adheres to the valves of the heart. The skin manifestations of infective carditis are thought to be result of septic emboli and immune-mediated reactions generated by chronic bacterial infection. Conjunctival, palatal petechiae, splinter haemorrhages, Osler nodes, painful erythematous nodules involving the pulp of the fingertips and toes are observed in patient suffering from bacterial endocarditis. ${ }^{3}$

\section{Acute Rheumatic fever}

Rheumatic fever is result from an autoimmune response in which antibodies directed against group A streptococcal antigen cross- react to similar antigens in human tissue. ${ }^{4}$

Financial or Other, Competing Interest: None.

Submission 23-11-2016, Peer Review 20-12-2016,

Acceptance 27-12-2016, Published 02-01-2017.

Corresponding Author:

Dr. Manabendra Nayak,

Lachit Nagar,

Raghunath Choudhury Path,

House No. 09,

Guwahati-781007, Assam, India.

E-mail:dr.mnayak@yahoo.co.in

DOI: $10.14260 /$ jemds $/ 2017 / 16$
Erythema marginatum occurs early in rheumatic fever and may persist after all other manifestations have resolved. It appears as non-pruritic, blanching, erythematous lesion with a raised serpiginous margin that involves the trunk and the proximal extremities while sparing the face. Individual lesions may appear and disappear within hours. The nodules are small, firm and painless and most commonly affected the tendons or bony surfaces, particularly the elbow.

\section{Cholesterol Embolization Syndrome}

Cholesterol embolization syndrome also known as blue toe syndrome is a multisystem disease resulting from the disruption of cholesterol crystals from atheromatous plaques on walls of the aorta or other arteries. Livedo reticularis, a red or blue, mottled, netlike discolouration, is a classic finding. Other findings include ulceration, cyanosis, purpura, necrosis, gangrene and painful nodules. Most cases involve the lower extremities and often bilateral. 5

\section{Lipid Disorders}

Disorders in Lipid metabolism due to genetic defects or secondary aetiologies result not only in the formation of distinct cutaneous lesion but may lead to life threatening atherosclerotic cardiovascular disease as well. Xanthomas, are localized lipid laden infiltrates in the dermis or tendons appearing as yellow - orange superficial papules, plaques or nodules. ${ }^{6}$ Planar xanthomas are soft yellow macules or plaques on the eye, neck, palms and chest. Xanthelasmas, the most common form of planer xanthoma, are small yellow papules often a few millimetres in diameter that involve the upper and lower eyelid. Tuberous xanthemas appear as painless yellow or red nodules that may reach a diameter of 5 $\mathrm{cm}$ or more and involve the elbow, hand, buttocks and heals. They are associated with familial dysbetalipidemia, familial combined hypertriglyceridemia and homozygous familial hypercholesterolemia. 


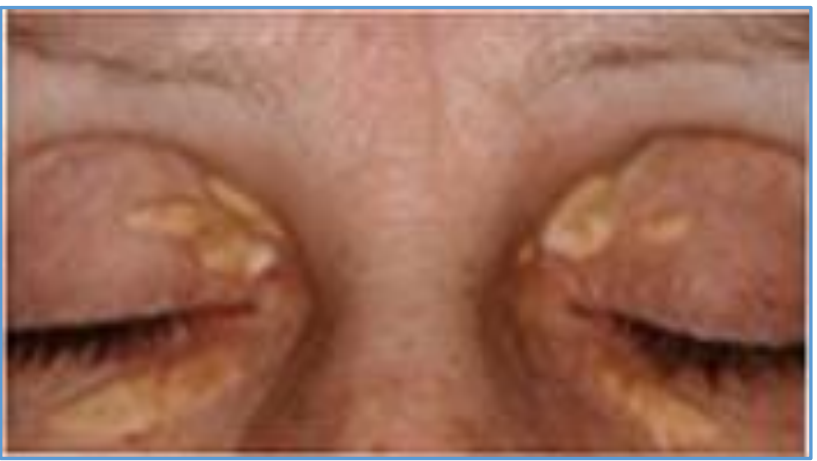

Figure 1. Xanthelasma

\section{Cardiac Myxomas}

Myxomas are the most common primary cardiac tumour, occurring with a higher frequency in women. Located most frequently in the left atrium, myxoma are large, sometimes friable pedunculated tumours with diameters as large 15 cms. ${ }^{7}$ Patients may present with nonspecific skin findings secondary to embolism of the myxoma including Raynaud phenomenon, petechiae, telangiectasia, splinter haemorrhages or livedo reticularis. Pruritic erythematous macules or papules may be observed on the extremities. ${ }^{8}$ In additions, painful, violaceous, serpiginous legions on the distal extremities may be present. Biopsy demonstrates intravascular myxomatous material.

\section{Leopard Syndrome}

Leopard syndrome is an autosomal dominant disorder of variable penetrance and expression caused by mutations of the neural ectoderm. The mnemonic describing the characteristics of the disorder was first used by gorlin et al. in 1969 and stands for lentigines. Electrocardiographic abnormalities, ocular telorism, pulmonary valve stenosis, abnormal genitalia, retardation of growth and deafness. ${ }^{9}$

The lentigines are dark brown irregularly shaped macules ranging in size from pinpoint to $5 \mathrm{~cm}$ with involvement of the face, neck and upper trunk. The extremities and genitalia are less commonly involved. Lentigines are present at birth or develop in childhood, becoming progressively darker and more numerous with age. Other cutaneous abnormalities have been reported in leopard syndrome including auxiliary freckling café au lait spots, localized hypopigmentation interdigital webs, dermatoglyphic abnormalities, onychodystrophy, multiple granular cell myoblastomas and hyperelasticity. ${ }^{10}$

\section{Cutaneous Manifestations of Renal Disease}

A wide variety of skin manifestation occurs in patients with chronic kidney diseases (CKD). Although majority of skin disorders in CKD are relatively benign, a few rare skin diseases have the potential to cause serious morbidity and mortality. Pico et al ${ }^{11}$ found that CKD patients, all had at least 1 form of skin alteration. Premature skin ageing makes these patients appear considerably older than their age. Patients are also prone to increased hair growth on their cheeks and thickening of the eyebrows. ${ }^{12}$

\section{Uremic Pruritus}

Pruritus remains an important health issue in patients with CKD, affecting patients on haemodialysis more frequently than those on peritoneal dialysis. ${ }^{13}$ Studies have shown that pruritus affects $50 \%-90 \%$ of patients with CKD, is independent of sex, age, race or aetiology of kidney failure ${ }^{13}$, and can be debilitating. Despite much speculation, the exact mechanism of pruritus remains unclear. Although xerosis is common in dialysis patients, it does not appear to correlate with severity of pruritic. CKD patients have excessive numbers of mast cell in the skins, and it has been proposed that histamine plays an important role in the development of pruritus. However, data to support the claims are lacking. The occurrence, duration and intensity of pruritus can change over time and the itching is usually worst at night. The areas most commonly affected are the back, limbs, chest and head, but $20 \%-50 \%$ of patients experience generalized pruritus. ${ }^{13}$ Uremic pruritus often leads to considerable mechanical skin damage as a result of continuous scratching with excoriations, super imposed infections and chronic lesions of prurigo nodularis or skin lichenification often occurring. Despite these findings, uremic pruritus remains and underappreciated complication that adversely affects the quality of life of many patients with CKD.

\section{Acquired Perforating Dermatosis (Kyrle's Disease)}

Kyrle's disease is a perforating skin disorder that occurs in upto $10 \%$ of CKD patients undergoing haemodialysis. ${ }^{14}$ It is usually characterized by a linear confluence of papules with a central, oyster shell- like keratotic plug on the trunk, proximal extremities, scalp and face. Lesions are red or pink in white patients, and hyperpigmented in black patients. 15 Origin of acquired perforating dermatosis lesion is not known, suspected causes include an inflammatory skin reaction secondary to the presence of uremic toxins, uric acid deposits or scratching induced trauma. ${ }^{15}$

- Hyperpigmentation.

- Pallor.

- Xerosis

- Ichthyosis.

- Pruritus.

- Prurigo nodularis.

- Acquired perforating dermatosis (Kyrle disease).

- Bacterial, fungal and viral infections.

- Purpura.

- Porphyria cutanea tarda.

- Pseudoporphyria.

- Calcific Uremic Arteriolopathy.

- Benign nodular calcification.

- Half- and - half nails.

- Onychomycosis.

- Splinter haemorrhages.

- Subungual hyperkeratosis.

- Brittle hair.

- Alopecia.

- $\quad$ Red eyes (pinguecullitis).

- Angular cheilitis.

- Uremic frost.

- Nephrogenic systemic fibrosis.

Table 1. Skin disorders in patients with renal disease 


\section{Nephrogenic Systemic Fibrosis (NSF)}

NSF previously known as nephrogenic fibrosing dermopathy, is a scleroderma - like fibrosing disorder that occurs in patients with CKD, renal transplant recipients and patient with acute kidney injury. The condition in characterized by painful and debilitating, progressive fibrosis and thickening of the skin, with occasional involvement of other organs and tissues such as the lungs, heart, liver, oesophagus, testes, dura mater and striated muscles. ${ }^{16}$

Patients with NSF initially complain of tightening and swelling of the skin of the lower and upper extremities, with light or dark red discoloration of the skin. In more advanced stages, flexion contractures of the joint occur, and mobility become severely compromised. Muscles weakness and joint contracture cause patients to become wheel chair - bound over time.

\section{Calcific Uremic Arteriolopathy (CUA)}

CUA or calciphylaxix, is a potentially life - threatening vasculopathy of the skin and subcutaneous tissues that is usually associated with CKD. Subcutaneous nodules are often palpable finally, the area becomes overtly necrotic with scar formation, and lesions may open and become infected. The histological lesion characteristically involves epidermal ulceration, dermal necrosis, and vascular medial wall calcifications, with subintimal hyperplasia and fibrosis of small and medium - sized blood vessels in the dermis and subcutaneous tissues. ${ }^{17}$

\section{Porphyria Cutanea Tarda (PCT)}

PCT in patients with CKD commonly presents as bullae on the dorsal surfaces of the hands and feet; bullae sometimes occur on the face as well, usually accompanied by facial hyperpigmentation and hypertrichosis. The sporadic form of PCT occurs in approximately $5 \%$ of patients in dialysis, this form is caused by increased uroporphyrin concentrations. ${ }^{18}$

\section{Cutaneous Manifestation of Internal Malignancy}

Many malignant conditions that effect internal organs display cutaneous manifestation, which may be presenting sign and symptoms of the underlying malignancy.

\section{Cowden Syndrome}

Cowden syndrome in an autosomal dominant condition. A variety of cutaneous and mucosal manifestation can occur, these are observed at any time form childhood to middle age. Papules are found mainly on the head and neck, hand and feet and my-assume a wart like appearance. Similar papules on the tongue and gingival may coalesce to produce a cobblestone appearance. ${ }^{19}$ Lipomas and haemangiomas may complete the clinical picture.

\section{Acanthosis Nigricans}

Acanthosis nigricans is perhaps the best for the cutaneous marker of internal malignancy. ${ }^{20}$ Affected skin has a hyperpigmented, velvety appearance and in severe cases can become quite verrucesus. Papillomatous changes may be noted in the oral cavity, and hyperkeratosis is a wrinkled or ridged pattern may develop on the palms and the dorsal surfaces of large joints. ${ }^{20}$ The cutaneous charges can occur before, coincident with, or after the discovery of an underlying malignancy, which most often is an adenocarcinoma of the stomach and other organs within the abdominal cavity.

\section{Pyoderma Gangrenosum}

Pyoderma Gangrenosum is a neutrophilic ulcerative dermatosis. Lesions appear as purulent ulcer, often with cyanotic overhanging borders. Occasionally classical pyoderma gangrenosum has been associated with a monoclonal gammopathy, with several solid tumours and with non- Hodgkin Lymphoma. The superficial form of pyoderma gangrenosum, known as atypical or bullous pyoderma gangrenosum. Most often, the association is with acute myelogenous leukaemia, but several cases of chronic myelogenous leukaemia, acute lymphoblastic leukaemia, myelofibrosis or agnogenic myeloid metaplasia have also been reported. The skin and blood disease often present concurrently and run a parallel course. ${ }^{21}$

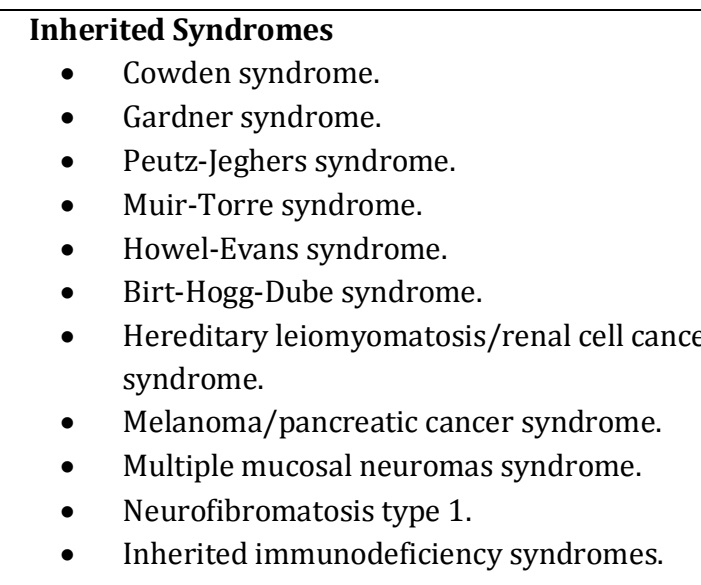

\section{Hormone-Secreting Tumours}

- $\quad$ Ectopic ACTH syndrome.

- Carcinoid syndrome.

- Multiple endocrine neoplasia syndrome.

- Glucagonoma syndrome.

\section{Proliferative and Inflammatory Dermatoses}

- Acquired hypertrichosis lanuginose.

- Acanthosis nigricans.

- Sign of Leser-Trelat.

- $\quad$ Triple palms.

- Bazex syndrome.

- Primary systemic amyloidosis.

- Scleromyxedema.

- Sweet syndrome.

- Pyoderma gangrenosum.

- Blistering disorders.

- Dermatomyositis.

- Clubbing and related disorders.

- Cutaneous leukocytoclastic vasculitis.

- Coagulopathies.

- Figurate erythemas.

- Extramammary Paget disease.

- Infectious disorders.

- Generalized pruritus, ichthyosis, and exfoliative dermatitis.

- Pigmentary disorders.

\section{Miscellaneous skin, hair, and nail disorders}

Table 2. Cutaneous Magnifestations of Internal Malignacy 


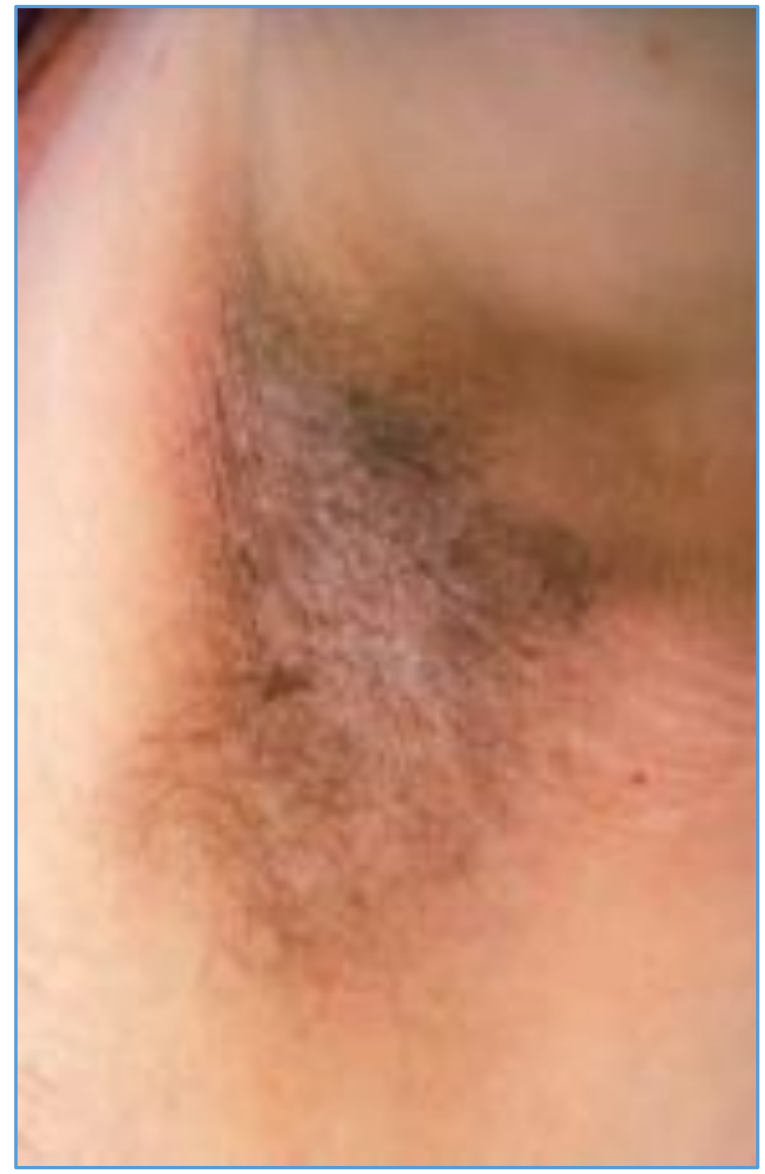

Figure 2. Acanthosis

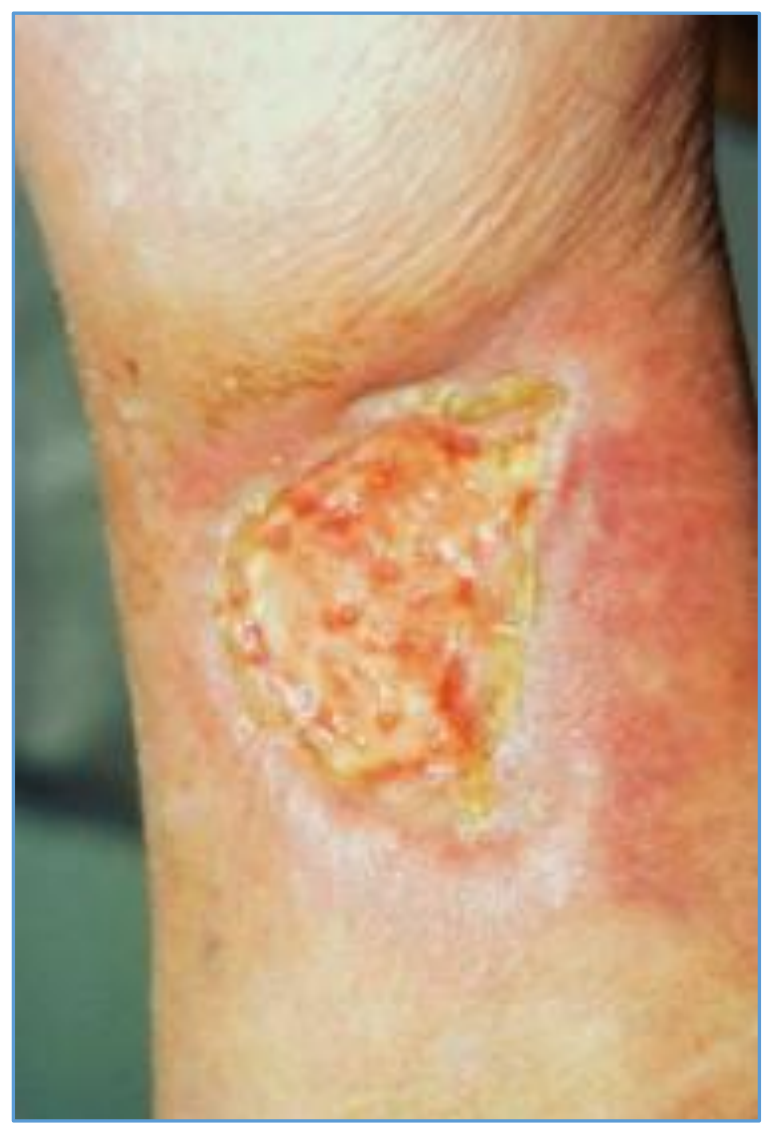

Figure 3. Pyoderma gangrenosum

\section{Dermatomyositis}

Approximately $10 \%-30 \%$ of adult patients with dermatomyositis have an associated malignancy. ${ }^{22}$ An awareness of this potential should alert the physicians to carefully evaluate all dermatomyositis patients for malignancy. Clinical manifestation of dermatomyositis includes as oedematous, violaceous eruption of the upper eyelids and atrophic scaly papules over bony prominences. Malar erythema, scalp inflammations, poikiloderma and periungual telangiectasia are significant, although less specific findings.

\section{Pigmentary Disorders}

Hypopigmentation or hyperpigmentation of the skin may be a sign of internal malignancy. The pigmentary changes may be idiopathic or related to the activity of a substance produced by the tumour. Ectopic ACTH production may stimulate cutaneous melanocytes, resulting in diffuse hyperpigmentation. Diffuse hyperpigmentation my also occur in hemochromatosis, liver cancer, and a widely metastatic melanoma.

\section{Acrokeratosis Paraneoplastica}

Acrokeratosis paraneoplastica was initially described as a skin marker for supradiaphragmatic neoplasia by Bozex in 1965, particularly squamous cell carcinoma of the upper aerodigestive tract. ${ }^{23}$ Dermatologic findings include erythematous to violaceous plaques - like scale, symmetrically distributed on the acral areas, ear helicos, and nasal and malar surfaces. In later stages the limbs and trunk become involved. Other cutaneous findings include hyperpigmentation, palmoplantar keratoderma and paronychia. ${ }^{24}$ The presence of acrokeratosis paraneoplastica should prompt a search for an internal malignancy and required a complete physical examination, including head, neck, and pelvic examinations together with appropriate laboratory, radiologic and endoscopic evaluation.

\section{Glucagonoma Syndrome and Necrolytic Migratory Erythema \\ Glucagonoma syndrome is a rare glucagon secreting pancreatic alpha cell tumour, associated with characteristic cutaneous findings known as necrolytic-migratory erythema (NME). NME, erythematous eruption with central blister leading to secondary erosions, crusting and subsequent hyperpigmentation. ${ }^{25}$ Lesion may become confluent and can wax and wane over a 7 to 10 days period. Perineum, ischial surfaces, groin and lower abdomens are favored locations, but NME can also occur in periorofacial distribution.}

\section{Carcinoid Syndrome}

Carcinoid syndrome has several stages of cutaneous involvement. Bright red flushing of the face, neck and upper aspect of the trunk lasting for 1-2 minutes is a characteristic cutaneous finding. ${ }^{26}$ Flushing can become more intensive, involving the entire trunk and extremities. Foregut tumours produce a Saloman pink/ bright red flushing, Midgut tumours produce a cyanotic flush, which is associated with classical carcinoid syndrome. Scleroderma is feature of carcinoid tumour arising in gut, is a poor prognostic indicator in patients with carcinoid syndrome and is often irreversible. 


\section{CONCLUSION}

Skin disorders are a common problem in patient with different internal diseases, and can affect the patients physical and mantel health and thus their quality of life. A basic knowledge of the most common dermatological entities encountered in different diseases will enable physicians to optimize daily patient care and to recognize potentially life threatening conditions.

\section{REFERENCES}

[1] Lee A. Skin manifestations of systemic disease. Australian Family Physician 2009;38(7):498-505.

[2] Tuppal R. Cutaneous manifestation of internal disease. The Canadian Journal of CME 2008:64-70.

[3] Uliasz A, Lebwohl M. Cutaneous manifestations of cardiovascular diseases. Clinics in Dermatology 2008;26(3):243-54.

[4] Dajani AS. Current status of non-supportive complications of group A streptococcus. Pediatir Infect Dis J 1991;10(10 Suppl):S25-7.

[5] Fukumoto Y, Tsutsui H, Tsuchihashi M, et al. The medicine and risk factors of cholesterol embolization syndrome, a complication of cardiac catheterization, a prospective study. J Am Coll Cardiol 2003;42(2):211-6.

[6] Russo GG. Hyperlipidemias. Clin Dermatol 1996;14(4):367-74.

[7] Vander Salm TJ. Unusual primary tumour of the heart. Semin Thoracic Cardiovasc Surg 2000;12(2):89-100.

[8] Gareia F-Villata MJ, Sanz-Sanchez T, Aragüés M, et al. Cutaneous embolization of cardiac myxoma. $\mathrm{Br} \mathrm{J}$ Dermatol 2002;147(2):379-82.

[9] Gorlin RJ, Anderson RC, Blow M. Multiple lentigines syndrome. Am J Dis Child 1969;117(6):652-62.

[10] Voron DA, Hatfield HH, Kalkhoff RK. Multiple lentigines syndrome. Case report and review of the literature. Am J Med 1976;60(3):447-56.

[11] Pico MR, Lugo-Somolinos A, Sánchez JL, et al. Cutaneous alterations in patients with chronic renal failure. Int J Dermatol 1992;31(12):860-3.

[12] Avermaete A, Altmeyer P, Bacharach-Buhles M. Skin changes in dialysis patients a review. Nephrol Dial Transplant 2001;16(12):2293-6.
[13] Lugon JR. Uremic pruritus: a review. Hemodial Int 2005;9(2):180-8.

[14] Knable AL. Cutaneous nephrology. Dermatol Clin 2002;20(3):513-21.

[15] Saray Y, Seçkin D, Bilezikçi B, et al. Acqired perforating dermatosis: clinicopathological features in twenty-two cases. J Eur Acad Dermatol Venereol 2006;20(6):67988.

[16] Menooza FA, Artlett CM, Sandorfi N, et al. Description of twelve cases of nephrogenic fibrosing dermopathy and review of the literature. Semin Arthrities Rheum 2006;35(4):238-49.

[17] Essary LR, Wick MR. Cutaneous calciphylaxis. An underrecognized clinicpathologic entity. Am J Clin Pathol 2000;113(2):280-7.

[18] Glynne P, Deacon A, Goldsmith D, et al. Bullous dermatoses in end stage renal failure; porphyria or pseudoporphyria? Am J Kidney Dis 1999;34(1):15560 .

[19] Hildenbrand C, Burgdorf WH, Lautenschlager S. Cowden syndrome-diagnostic skin signs. Dermatology 2001;202(4):362-6.

[20] Anderson SH, Hudson-Peacock M, Muller AF. Malignant acanthosis nigricans: potential role of chemotherapy. Br J Dermatol 1999;141(4):714-6.

[21] Callen JP, Jackson JM. Pyoderma gangrenosun: an update. Rheum Dis Clin North Am 2007;33(4):787802.

[22] Chen YJ, Wu CY, Shen JL. Predicting factors of malignancy in dermatomyositis and polymyositis: a case-control study. Br J Dermatol 2001;144(4):82531.

[23] Sharma V, Sharma NL, Ranjan N, et al. Acrokeratosis paraneoplastica. Case report and review of literature. Dermatol Online J 2006;12(1):11.

[24] Bolognia JL. Bazex syndrome: acrokeratosis paraneoplastica. Semin Dermatol 1995;14(2):84-9.

[25] Technau K, Renkl A, Norgauer J, et al. Necrolytic migratory erythema and myelodysplastic syndrome without glucogonoma. Eur J Dermatol 2005;15(2):110-2.

[26] Braverman IM. Skin manifestation of internal malignancy. Clin Geriatr Med 2002;18(1):1-19. 